\title{
Preparation of Boron Nitride Nanoplatelets via Amino Acid Assisted Ball Milling: Towards Thermal Conductivity Application
}

\author{
Nan Yang ${ }^{1, \dagger}{ }^{+}$Haifeng Ji ${ }^{1, \dagger}$, Xiaoxia Jiang ${ }^{2}$, Xiongwei Qu ${ }^{1}$, Xiaojie Zhang ${ }^{1, *}$, Yue Zhang ${ }^{1, *}$ and \\ Binyuan Liu ${ }^{1, *}$ \\ 1 Hebei Key Laboratory of Functional Polymers, Department of Polymer Materials and Engineering, \\ Hebei University of Technology, 8 Guangrong Street, Tianjin 300130, China; \\ 201721504016@stu.hebut.edu.cn (N.Y.); 201911501019@stu.hebut.edu.cn (H.J.); xwqu@hebut.edu.cn (X.Q.) \\ 2 School of Chemical Engineering and Technology, Hebei University of Technology, 8 Guangrong Street, \\ Tianjin 300130, China; jiangxx@hebut.edu.cn \\ * Correspondence: zhangxj@hebut.edu.cn (X.Z.); zylizzy@hebut.edu.cn (Y.Z.); byliu@hebut.edu.cn (B.L.); \\ Tel.: +86-13843143643 (X.Z.) \\ + These authors contributed equally to this work.
}

Received: 11 August 2020; Accepted: 21 August 2020; Published: 22 August 2020

\begin{abstract}
Hexagonal boron nitride nanoplatelets (BNNPs) have attracted widespread attention due to their unique physical properties and their peeling from the base material. Mechanical exfoliation is a simple, scalable approach to produce single-layer or few-layer BNNPs. In this work, two amino acid grafted boron nitride nanoplatelets, Lys@BNNP and Glu@BNNP, were successfully prepared via ball milling of h-BN with L-Lysine and L-Glutamic acid, respectively. It was found that the dispersion state of Lys@BNNP and Glu@BNNP in water had been effectively stabilized due to the introduction of amino acid moieties which contained a hydrophilic carboxyl group. PVA hydrogel composites with Lys@BNNP and Glu@BNNP as functional fillers were constructed and extensively studied. With 11.3 wt \% Lys@BNNP incorporated, the thermal conductivity of Lys@BNNP/PVA hydrogel composite was up to $0.91 \mathrm{~W} \mathrm{~m}^{-1} \mathrm{~K}^{-1}$, increased by $78 \%$, comparing to the neat PVA hydrogel. Meanwhile, the mechanical and self-healing properties of the composites were simultaneously largely enhanced.
\end{abstract}

Keywords: amino acids; hexagonal boron nitride nanoplatelets; mechanical exfoliation; self-healing; thermal conductivity

\section{Introduction}

Hexagonal boron nitride nanoplatelets (BNNPs), which feature a layered structure analogous to graphene [1-3], have gained increasing attention, as they share many of the advantageous properties of graphene, but also show other excellent properties, including highly thermal and chemical stability [4-6], excellent mechanical strength [7], and high thermal conductivity [8]. BNNPs consist of $\mathrm{sp}^{2}$-bonded atomic layers where $\mathrm{B}$ and $\mathrm{N}$ atoms are alternately arranged into a hexagonal lattice. The regular atomic arrangement permits high phonon velocity and low phonon scattering, leading to an excellent thermal conductive property $\left(\sim 2000 \mathrm{~W} \mathrm{~m}^{-1} \mathrm{~K}^{-1}\right)$ [9]. Compared to graphene, unique insulating properties make BNNPs especially promising in thermally conductive yet electrically insulating polymer composites $[10,11]$. However, the preparation of BNNPs still faces a lot of challenges, such as high energy consumption, low yields of production and poor repeatability $[12,13]$. Recently, some improved methods have been reported to produce BNNPs by exfoliation of hexagonal boron nitride (h-BN) via mechanical cleavage [14]. Up to now, a large number of methods have been explored for the exfoliation process of $\mathrm{h}-\mathrm{BN}$. For example, 
Lin [15] et al. have reported that ball-milled h-BN, where defects had been introduced intentionally, could be further functionalized with a long alkyl chain amine. Chen [16] and his colleagues present a simple and efficient one-step method for the preparation of functionalized few-layer BNNPs by ball milling of commercially available h-BN and urea powder. Among the various mixtures of methods, amino groups play a critical role, since they can produce Lewis acid-base interactions with the electron-deficient boron atoms on the h-BN surface or defects during ball milling [17,18].

Recently, amino acids (AAs) have attracted considerable interest because they have the advantage of origin from biomass sources and are cheap and readily available $[19,20]$. Owing to their multifunctional groups, AAs have also been widely used in constructing polymer materials with various properties [21,22]. Considering the inherent existence of amino groups, and inspired by the unique structure of AAs, which consist of amino and carboxyl groups and various R-side-chains, we proposed that we could employ the AAs as the ball-milling reagent to exfoliate h-BN. With this approach, h-BN could be exfoliated to BNNPs via Lewis acid-base interactions between the amino groups and boron atoms on the surface of BNNPs, meanwhile carbon-chains, carboxyl and different functional R-side-chains could be grafted onto BNNPs simultaneously. The introduction of amino acids can not only improve the compatibility between the BNNPs and the polymer substrate owing to the carbon-chain moieties, but also endow BNNPs with multi-functions, such as hydrophilicity and hydrogen-bonding interactions, which are attributed to the presence of carboxyl groups and various R-side-chains.

Herein, we made a preliminary attempt to discuss the feasibility for improved mechanical exfoliation of h-BN by using an L-Lysine and L-Glutamic acid-assisted ball-milling process, forming Lys@BNNP and Glu@BNNP. The chemical structures of both Lys@BNNP and Glu@BNNP were proved by specific structure characterization, such as FT-IR and XPS. The results of TEM, SEM and XRD showed that BNNPs with a thinner layer thickness than that of h-BN were obtained, which indicated successful exfoliation. We believe that amino acid-assisted ball-milling exfoliation of boron nitride provides a low-cost, effective and easy-to-operate method for the preparation of multifunctional BNNPs. Furthermore, PVA hydrogel composites with Lys@BNNP and Glu@BNNP as functional fillers have been constructed and studied for highly thermal conductivity and mechanical properties.

\section{Materials and Methods}

\subsection{Materials}

L-Lysine, L-Glutamic acid and PVA (1799) were purchased from Aladdin reagent Co., Ltd. (Shanghai, China). Hexagonal boron nitride (h-BN) with lateral particle size in the range of 3-5 $\mu \mathrm{m}$ was purchased from Shandong Qingzhou Matekechuang Materials Co., Ltd. (Shandong, China) Sodium hydroxide, borax and ethanol were purchased from Fuchen Chemical Reagent Co., Ltd. (Tianjin, China). $\mathrm{HCl}(37 \%)$ were provided by Tianjin keraisi Fine Chemical Co., Ltd. (Tianjin, China).

\subsection{Experiment}

\subsubsection{Exfoliation of BNNP by $\mathrm{H}_{2} \mathrm{O}$}

A high energy ball mill (JX-5G) was utilized for mechanical exfoliation. At room temperature, $40 \mathrm{~g}$ of micron-sized h-BN powder (used as received) and $80 \mathrm{~g} \mathrm{H}_{2} \mathrm{O}$ were loaded into a $3 \mathrm{~L}$ ball mill tank with $2 \mathrm{~kg}$ zirconia ball bead $(8 \mathrm{~mm}: 5 \mathrm{~mm}: 3 \mathrm{~mm}=1: 2: 1)$. The rotational speed of the energy ball mill was set to $350 \mathrm{rpm}$, and the mixture was milled for $10 \mathrm{~h}$. After cooling back to room temperature, the exfoliation product was collected and rinsed with large amounts of deionized water and ethanol. Followed by filtration and drying in a vacuum oven $\left(60^{\circ} \mathrm{C}\right)$, BNNP was obtained as a white powder with a yield of $92 \%$.

\subsubsection{Exfoliation of h-BN by L-Lysine Assisted Ball Milling (Lys@BNNP)}

A high energy ball mill (JX-5G) was utilized for exfoliation. At room temperature, $40 \mathrm{~g}$ of micron-sized h-BN powder (used as received), $80 \mathrm{~g}$ of L-Lysine and $365 \mathrm{~mL}$ of $1.5 \mathrm{M} \mathrm{NaOH}$ solution 
(for the protection of the ball milling equipment) were loaded into a $3 \mathrm{~L}$ ball mill tank with $2 \mathrm{~kg}$ zirconia ball beads $(8 \mathrm{~mm}: 5 \mathrm{~mm}: 3 \mathrm{~mm}=1: 2: 1)$. The rotational speed of the energy ball mill was set to $350 \mathrm{rpm}$, and the mixture was milled for $10 \mathrm{~h}$. After cooling back to room temperature, the exfoliation product was collected and rinsed with large amounts of deionized water and ethanol. Followed by filtration and drying in a vacuum oven $\left(60^{\circ} \mathrm{C}\right)$, Lys@BNNP was obtained as a white powder in a yield of $85 \%$.

\subsubsection{Exfoliation of h-BN by L-Glutamic Acid Assisted Ball Milling (Glu@BNNP)}

The procedure for the synthesis of Lys@BNNP was followed to prepare Glu@BNNP from h-BN and L-Glutamic acid as a white powder with a yield of $83 \%$.

\subsubsection{Preparation of Pure PVA Hydrogel}

The route to PVA hydrogel is similar to the previous work [23]. In this experiment, PVA (1799) powder was first dissolved in hot water $\left(98^{\circ} \mathrm{C}\right)$ to form a $12.5 \mathrm{wt} \%$ transparent solution. Using borax as a crosslink reagent, PVA/borax solution (40 mL PVA solution and $15 \mathrm{~mL} 0.06 \mathrm{M}$ borax aqueous solution) was stirred in ultrasonic dispersion for $1 \mathrm{~h}$. Finally, the PVA hydrogel was placed in a cylindrical mold, and a heavy object $(\sim 5.0 \mathrm{~kg})$ was placed on the surface of the hydrogel. The purpose of pressing with a heavy object was to remove bubbles and repair cracks in the hydrogel.

\subsubsection{Preparation of h-BN/PVA, BNNP/PVA, Lys@BNNP/PVA and Glu@BNNP/PVA Hydrogels}

In this experiment, PVA powder was first dissolved in hot water $\left(98^{\circ} \mathrm{C}\right)$ to form a $12.5 \mathrm{wt} \%$ transparent solution. Using borax as crosslink reagent, PVA/borax solution (40 mL PVA solution and $15 \mathrm{~mL} 0.06 \mathrm{M}$ borax aqueous solution) was mixed with a designated amount of h-BN, BNNP/PVA, Lys@BNNP and Glu@BNNP, respectively, under stirring ultrasonic dispersion for $1 \mathrm{~h}$. Finally, the obtained hydrogels were placed in a cylindrical mold and a $5.0 \mathrm{~kg}$ pressure was applied for $2 \mathrm{~h}$ to remove the interior bubbles.

\subsection{Characterization}

\subsubsection{Structural Characterizations}

Fourier transform infrared spectroscopy (FT-IR) spectroscopy of h-BN, BNNP, Lys@BNNP and Glu@BNNP were recorded on a Vector-22 (Brucker, Germany) spectrometer. The spectral range was 400 to $4000 \mathrm{~cm}^{-1}$ with a resolution of $4 \mathrm{~cm}^{-1}$. All spectra were modified with carbon removal and baseline correction. Thermal gravimetric analysis (TGA) was conducted to evaluate the grafting content by using a SDT-TG Q600 thermogravimetric analyzer (TA Instruments, New Castle, DE, USA). The nitrogen flow rate was $100 \mathrm{~mL} \mathrm{~min}{ }^{-1}$, the heating rate was $10{ }^{\circ} \mathrm{C} \mathrm{min}^{-1}$, and the temperature scanning range was $100-700{ }^{\circ} \mathrm{C}$. X-ray diffraction (XRD) was used to study the crystallinity degrees of the h-BN, BNNP, Lys@BNNP and Glu@BNNP. XRD data was recorded on a D8 Advance (Brook AXS, Germany) X-ray diffractometer. The elemental composition of Lys@BNNP and Glu@BNNP was analyzed by ESCALAB 250Xi (Thermofisher Scientific, Waltham, MA, USA) X-ray photoelectron spectroscopy (XPS). The morphologies of h-BN, BNNP, Lys@BNNP and Glu@BNNP were observed by a Nova Nano SEM 450 (FEI, Hillsboro, OR, USA) scanning electron microscope (SEM). The morphology of the samples was investigated using transmission electron microscopy (TEM) (FEI Talos F200S, Hillsboro, OR, USA). Elemental mapping analysis was conducted by using a FEI Talos F200S microscope.

\subsubsection{Performance Characterizations}

The mechanical properties of the nanocomposites were characterized with a dynamic mechanical analyzer (DMA2000B, Tritec Instruments, York, UK) at an oscillation frequency of $1 \mathrm{~Hz}$. The rectangular samples $\left(35 \mathrm{~mm} \times 14 \mathrm{~mm} \times 4 \mathrm{~mm}\right.$ ) were cooled to $-50{ }^{\circ} \mathrm{C}$ under liquid nitrogen first, followed by heating up to $70{ }^{\circ} \mathrm{C}$ at the rate of $2 \mathrm{~K} \mathrm{~min}^{-1}$. 
Thermal conductivity test: The thermal conductivity of all PVA hydrogel samples were measured using TC 3000 Series Thermal Conductivity Apparatus (Xi'an Xiatech Electronic Technology Co., Xi'an, China) by a transient hot-wire technique. In this experiment, a thin metallic wire, as a line heat source and temperature sensor, is suspended between two identical hydrogel samples $(6.5 \mathrm{~cm} \times 3.5 \mathrm{~cm} \times 0.3 \mathrm{~cm})$. When the wire temperature is raised by a constant heat flux, it conducts heat to the surrounding medium, i.e., testing the samples. The rate of heat transfer associated with the thermal conductivity of the surrounding materials is reflected by the temperature changes of the wire [24]. Thus, the thermal conductivity $\lambda$ of the tested hydrogels can be calculated by the following formula:

$$
\lambda=\frac{q}{4 \pi(\mathrm{d} \Delta T / \mathrm{d} \ln t)}
$$

where, $q$ is the heat generation per unit time per unit length of the wire, $\Delta T$ is the temperature change of the wire, and $t$ is the testing time.

Self-Healing Experiments: Hydrogel splines were prepared on a template of $10 \mathrm{~cm} \times 1 \mathrm{~cm} \times 0.4 \mathrm{~cm}$. During the healing process, there was no other stress or outside stimulus applied. After self-healing, the tensile test was carried out again to calculate the healing efficiency [25].

Mechanical Measurements: The mechanical properties of hydrogels were determined by CMT6104 microcomputer controlled electronic universal testing machine. The gravitational sensor of the drawing machine was $100 \mathrm{~N}$. The hydrogel spline was prepared on the template of $10 \mathrm{~cm} \times 1 \mathrm{~cm} \times 0.4 \mathrm{~cm}$. In order to test the rapid self-healing performance of the BN samples, self-healing tests were carried out directly on the testing machine. Firstly, the spline was cut into halves, and then the two separate parts were re-contacted softly without other stress or outside stimulus applied. After waiting for predetermined seconds, the tensile test was carried out immediately. In this case, the healing time was determined from the re-contact to the pressing of the start button. The tensile rate was $100 \mathrm{~mm} \mathrm{~min}^{-1}$, and the initial gauge distance was $60 \mathrm{~mm}$. The data were the average strengths of five samples in the same group [23].

\section{Results and Discussion}

\subsection{Characterizations of $A A @ B N N P S$}

Figure 1 illustrates the overall procedure of the preparation of (amino acid)- $g$-BNNP hybrids (AA@BNNPs) and related AA@BNNP/PVA hydrogels. The Lys@BNNP and Glu@BNNP were prepared via ball milling with L-Lysine and L-Glutamic acid as assisting reagent, respectively, and fully characterized by FT-IR, TGA, XRD, TEM and XPS.

The structural differences of the raw h-BN and the AA@BNNPs were characterized by FT-IR, TGA, XRD and XPS. Figure 2a shows the FT-IR spectra, compared with h-BN, besides B-N bending and stretching vibrations $\left(817 \mathrm{~cm}^{-1}\right.$ and $\left.1378 \mathrm{~cm}^{-1}\right)$ [26,27], the spectra of BNNP exhibited new absorption bands around 3400 3600 $\mathrm{cm}^{-1}$, corresponding to O-H, and the spectra of AA@BNNPs exhibited several new absorption bands around $3400 \sim 3600 \mathrm{~cm}^{-1}, 2848 \mathrm{~cm}^{-1}$ and $2917 \mathrm{~cm}^{-1}$, and $1300 \sim 1500 \mathrm{~cm}^{-1}$, corresponding to $\mathrm{O}-\mathrm{H}$ and $\mathrm{N}-\mathrm{H}, \mathrm{C}-\mathrm{H}$, and $\mathrm{C}=\mathrm{O}$ stretching vibrations, respectively. This result indicated that amino acid moieties had been successfully grafted onto the surface of $h-B N$, since the unreacted amino acid was removed by repeated washing with DI water and ethanol. TGA measurements provided further evidence of the functionalization of BNNP and AA@BNNPs as shown in Figure $2 \mathrm{~b}$. For the Glu@BNNP and Lys@BNNP, a slight mass loss (1.0\% and 3.1\%) was observed, which was due to the removal of the grafted amino acid moieties. This meant that the grafted quantity of AA@BNNPs was correlating with the number of amino groups. As for BNNP, the mass loss was less ( $0.4 \%)$ due to the removal of hydroxyl groups. 


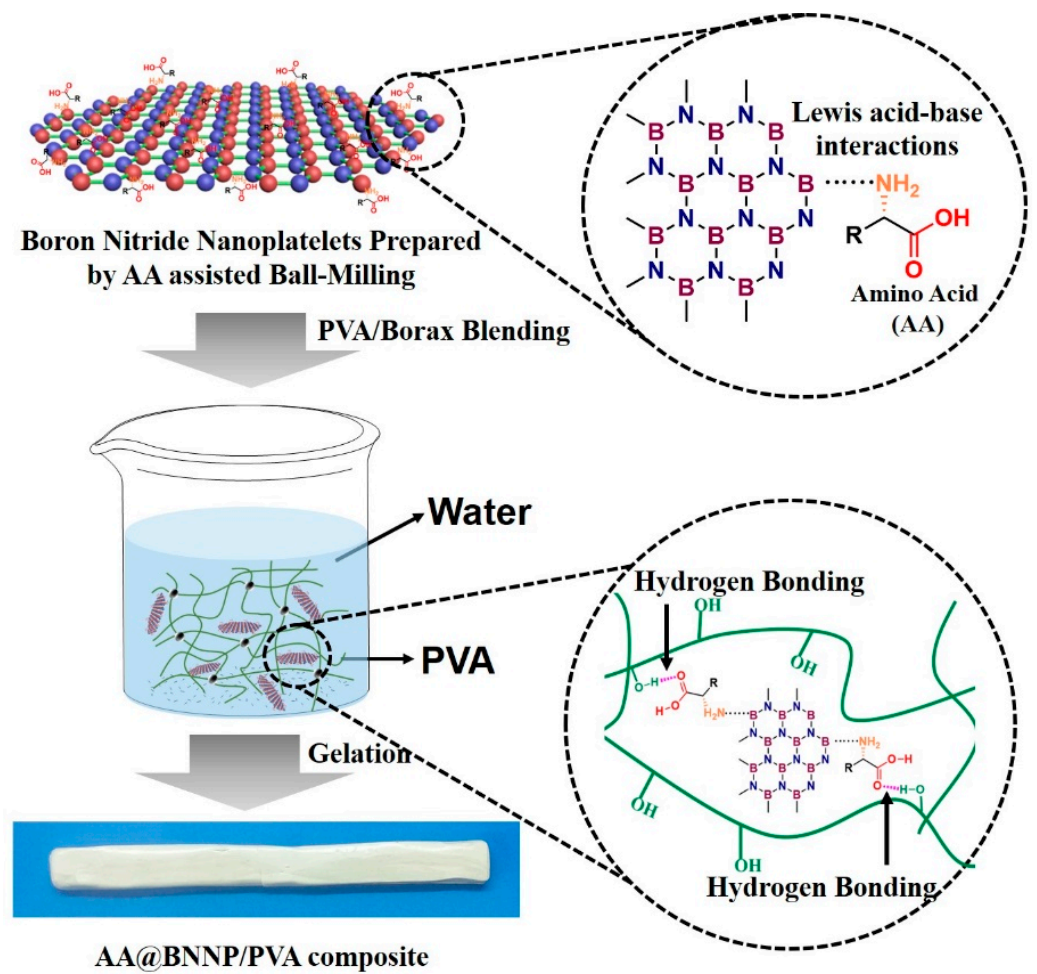

Figure 1. Schematic diagram showing the overall process for preparing the amino acid boron nitride nanoplatelet (AA@BNNP) composites.

The X-ray diffraction (XRD) of h-BN, BNNP, Lys@BNNP and Glu@BNNP powders is presented in Figure 2c. Two characteristic diffraction peaks were observed at $26.7^{\circ}$ and $42.1^{\circ}$ arising from (002) and (100) planes of the h-BN, respectively [16]. Apparently, the amino acid does not change the crystal structure of h-BN. A sharp (002) peak with considerably higher intensity at $2 \theta=26.7^{\circ}$ was presented in the XRD curve for h-BN. Moreover, the (002) peak of Lys@BNNP and Glu@BNNP showed a markedly reduced intensity and dramatically broadened width which indicated thinner BN layers in the hybrids [28]. The chemical bonding state of the AA@BNNPs was examined using X-ray photoelectron spectroscopy (XPS) as shown in Figure 2d,e. Four obvious peaks were observed in the XPS survey scans of Lys@BNNP and Glu@BNNP, corresponding to O 1s, N 1s, C 1s, and B 1s energy, respectively $[11,29]$. The B 1s spectra of Lys@BNNP and Glu@BNNP can be quantitatively differentiated into two different boron species. The strong peaks at 190.1 and $190.2 \mathrm{eV}$ were coming from the B-N bond, and the weak peaks at 191.0 and $190.8 \mathrm{eV}$ were ascribed to the B-O bond $[11,15,29]$. The N 1s spectra of Lys@BNNP and Glu@BNNP can be quantitatively differentiated into three different nitrogen species, which are C-N, B-N and B $\cdots \mathrm{N}[18,19,26,27,30]$. Notably, the emergence of new peaks of $C$ 1s and $O 1$ s were also observed with respect to amino acid moieties, in Figure S1. This observation also indicated that the AAs were successfully grafted onto the surface and edge of AA@BNNPs.

It is recognized that the size of the BNNPs has a strong influence on the mechanical and thermal properties of their macroscopic materials. Scanning electron microscopy (SEM) was employed for a detailed morphology study, as shown in Figure 3a-d. The large size of the raw h-BN material was clearly revealed from SEM observation. The raw h-BN sample showed a smooth surface and the layer thickness was around 200-300 nm (Figure 3a). After ball milling, the thickness of BNNP flakes decreased. Moreover, after fully exfoliating with AAs, there was a distinct change in the BN surface, the thickness of AA@BNNPs decreased to around $90 \mathrm{~nm}$, and the surface became rougher. This result was consistent with the XRD observation. 

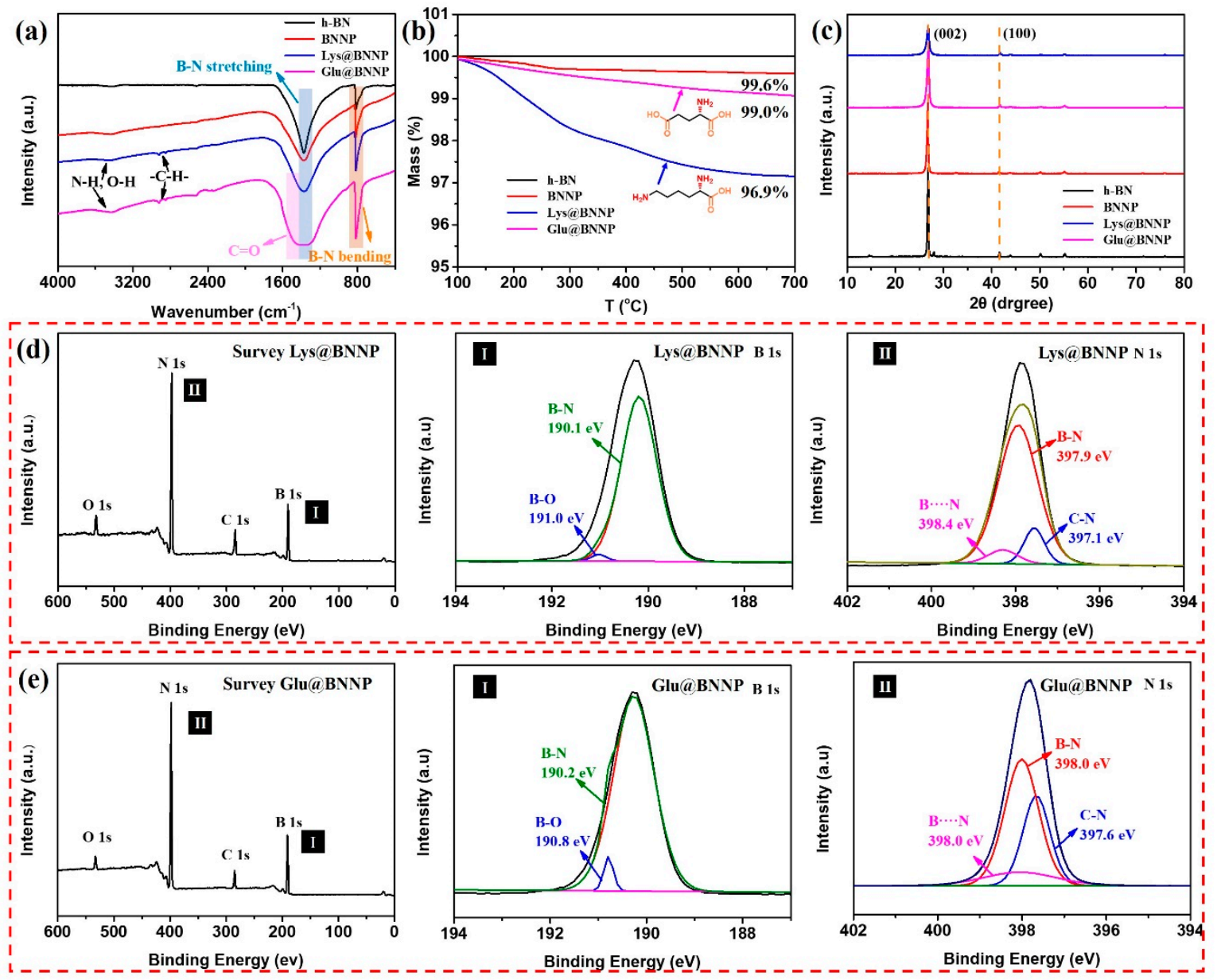

Figure 2. (a) FT-IR spectra of h-BN, BNNP, Lys@BNNP and Glu@BNNP; (b) TGA curves of h-BN, BNNP, Lys@BNNP and Glu@BNNP under a nitrogen flow; (c) XRD patterns of h-BN, BNNP, Lys@BNNP and Glu@BNNP powders; (d) high resolution XPS spectra of as-received Lys@BNNP, with (I) the B1s XPS spectra of the Lys@BNNP and (II) the O1s of the Lys@BNNP; (e) high resolution XPS spectra of as-received Glu@BNNP, with (I) the B1s XPS spectra of the Glu@BNNP and (II) the O1s of the Glu@BNNP.

Transmission electron microscopy (TEM) was used to observe the microstructure and crystallinity of BNNP, Lys@BNNP and Glu@BNNP. In Figure 3e,f and Figure S2, TEM images indicated that the basal plane of the AA@BNNPs remained intact, the structure of the few-layer BN remained ordered after ball milling and suggested that the hexagonal lattices of the AA@BNNPs were not damaged during the exfoliation processes. These results were also confirmed through the Raman spectra shown in Figure S3. A typical Raman signature was found for bulk h-BNs and exfoliated AA@BNNPs as a prominent peak in the region of $1364-1366 \mathrm{~cm}^{-1}$. It was seen that the peak intensity of BNNPs was notably decreased compared to its raw material h-BN, indicating a reduction of the weaker interaction between layers and layer numbers [9]. The amino acid-assisted exfoliation had not broken up or created any large holes in the nanoplatelets. Besides, compared to BNNP (Figure S2), the TEM results showed that in Glu@BNNP and Lys@BNNP the lamellae were flat and quite thin, which further demonstrated the successful exfoliation of Glu@BNNP and Lys@BNNP from bulk h-BN. It has been noted that the larger lateral size of the as-prepared BNNP was beneficial for effective thermal and mechanical reinforcements [31]. The presence of thick AA@BNNPs flakes can assist thermal transport in the composite because they are less subject to thermal conductivity degradation due to phonon-boundary scattering, and in the composite h-BN nanosheets can form thermal links that interconnect the thick h-BN flakes [32]. 

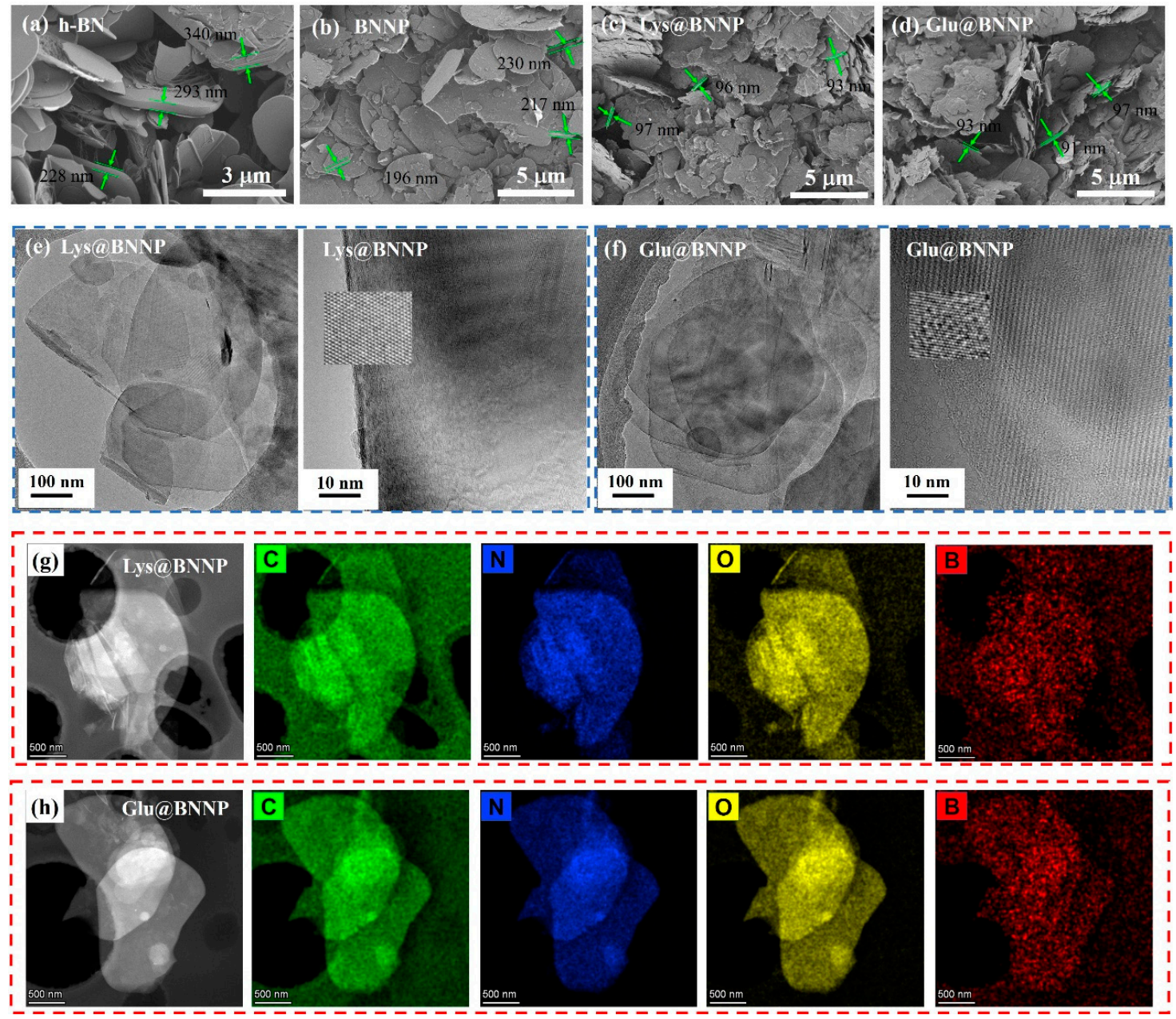

Figure 3. SEM images of (a) h-BN; (b) BNNP; (c) ball milling of Lys@BNNP and (d) ball milling of Glu@BNNP; (e) low-magnification TEM image of Lys@BNNP and high-resolution TEM images of the Lys@BNNP; (f) low-magnification TEM image of Glu@BNNP and high-resolution TEM images of the Glu@BNNP; high-magnification elemental mapping of (g) Lys@BNNP and (h) Glu@BNNP.

Elemental mapping of Lys@BNNP and Glu@BNNP are shown in Figure 3g,h. C, N, O and B elements were observed on the surface and edge of Lys@BNNP and Glu@BNNP samples, which further demonstrated the effective grafting and confirmed the good distribution of amino acid.

To further evaluate the applications of AA@BNNPs, we investigated the stability of the suspensions in water with different concentrations of h-BN, BNNP, Lys@BNNP and Glu@BNNP contents. Portions of $3 \mathrm{mg}, 6 \mathrm{mg}$ and $9 \mathrm{mg}$ of each BN samples were mixed with $10 \mathrm{~mL}$ water thoroughly in a vortex shaker for $1 \mathrm{~min}$ at room temperature. As observed in Figure $4 \mathrm{a}-\mathrm{d}$, after sitting for $24 \mathrm{~h}$, the untreated h-BN and BNNP dispersion either floated on the surface or sank to the bottom. The Lys@BNNP and Glu@BNNP dispersions with different concentrations $\left(0.3,0.6,0.9 \mathrm{mg} \mathrm{mL}^{-1}\right)$ remained stable and uniform. These results demonstrated that, after grafting of amino acid moieties, the hydrophilic carboxyl groups originating from AAs can help to stabilize the AA@BNNPs flakes in water [10,32]. This characteristic should be of benefit for potential applications of AA@BNNPs in constructing well-distributed hydrogels. 

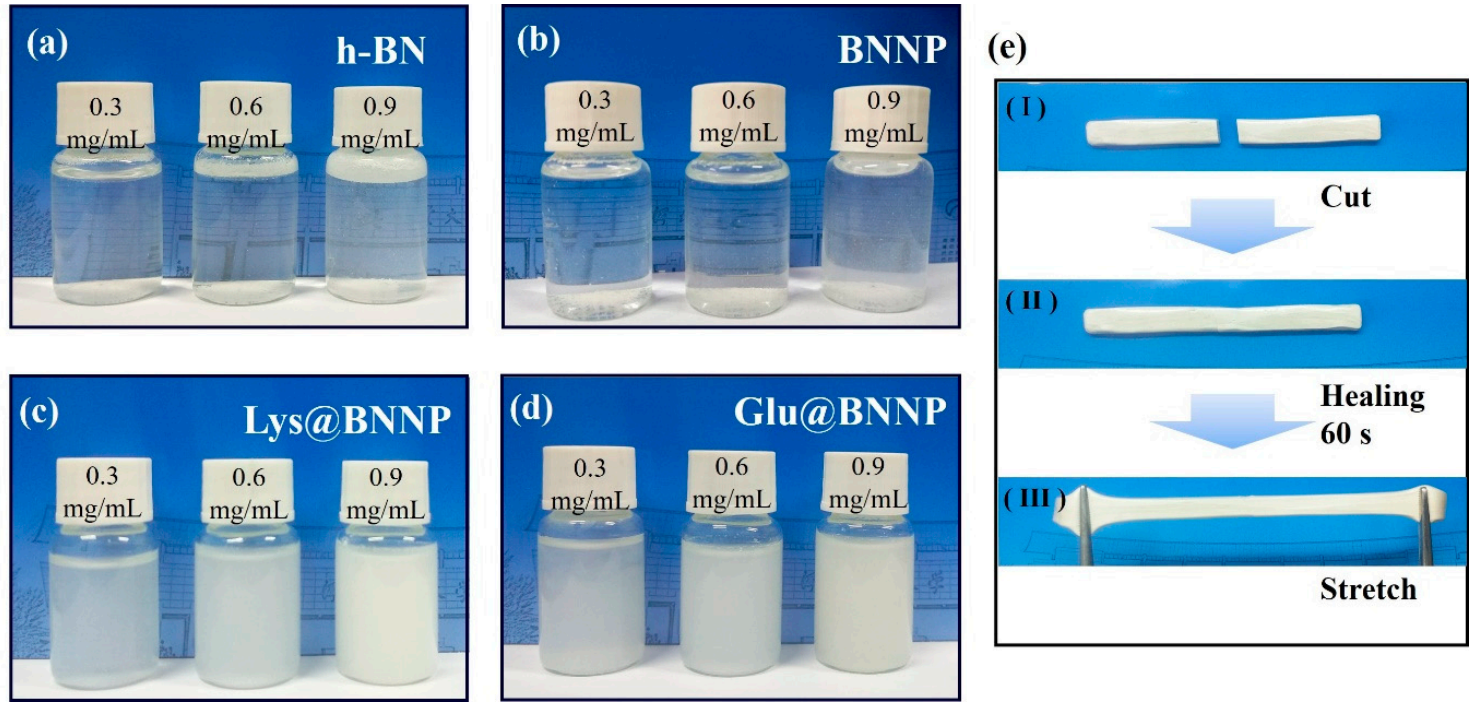

Figure 4. Photographs of as-prepared (a) h-BN; (b) BNNP; (c) Lys@BNNP and (d) Glu@BNNP dispersions after $24 \mathrm{~h}$ in a concentration of $0.3,0.6$ and $0.9 \mathrm{mg} \mathrm{mL}^{-1}$; (e) strip-shaped Lys@BNNP/PVA hydrogel with $11.2 \mathrm{wt} \%$ of Lys@BNNP: (I) the hydrogel is cut into two pieces; (II) the hydrogel can heal automatically after re-contacting for $60 \mathrm{~s}$ at room temperature; and (III) the healed hydrogel is stretched.

\subsection{Measurements of PVA Hydrogel}

PVA hydrogel composites with h-BN, BNNP, Lys@BNNP and Glu@BNNP as functional fillers have been constructed and studied for highly thermal conductivities, mechanical properties and self-healing behaviors. Figure 5a shows that the thermal conductivities of the PVA hydrogel composites gradually increased with the increase of filler content, with respect to the pure PVA hydrogel $\left(0.51 \mathrm{~W} \mathrm{~m}^{-1} \mathrm{~K}^{-1}\right)$. It was found that with $11.3 \mathrm{wt} \% \mathrm{AA} @ \mathrm{BNNPs}$ hybrids incorporated, the thermal conductivity of the Lys@BNNP/PVA hydrogel composite was up to $0.91 \mathrm{~W} \mathrm{~m}^{-1} \mathrm{~K}^{-1}$, increased by $78 \%$ comparing to the neat PVA hydrogel, as well as Glu@BNNP/PVA with a result of $0.87 \mathrm{~W} \mathrm{~m}^{-1} \mathrm{~K}^{-1}$. Notably, the filling amount of h-BN and BNNP in h-BN/PVA and BNNP/PVA hydrogels cannot reach $11.3 \mathrm{wt} \%$ due to its bad dispersion stability in water, as shown in Figure $4 \mathrm{a}, \mathrm{b}$. With a similar filling amount $(8.3 \mathrm{wt} \%)$, Lys@BNNP/PVA $\left(0.83 \mathrm{~W} \mathrm{~m}^{-1} \mathrm{~K}^{-1}\right)$ and Glu@BNNP/PVA $\left(0.82 \mathrm{~W} \mathrm{~m}^{-1} \mathrm{~K}^{-1}\right)$ also exhibited a higher thermal conductivity than that of h-BN/PVA $\left(0.76 \mathrm{~W} \mathrm{~m}^{-1} \mathrm{~K}^{-1}\right)$ and BNNP/PVA $\left(0.77 \mathrm{~W} \mathrm{~m}^{-1} \mathrm{~K}^{-1}\right)$, due to the thick AA@BNNPs flakes which assist thermal transport in the composite because they are less subject to thermal conductivity degradation due to phonon-boundary scattering. In the composite h-BN, nanosheets can form thermal links that interconnect the thick h-BN flakes [9], and the hydrogen-bonding interaction between $-\mathrm{OH}$ groups on the PVA chain and $-\mathrm{COOH}$ groups origin from amino acid grafts, leading to a better compatibility between the PVA matrix and AA@BNNPs [10,33].

In order to confirm the hydrogen-bonding interaction between - $\mathrm{OH}$ groups on PVA chain and $-\mathrm{COOH}$ groups, we investigated storage modulus (E) curves and loss factor $(\tan \delta)$ curves of the PVA composites by dynamic mechanical analysis (DMA) [11]. The mechanical properties of the composites were enhanced by improving the interfacial interaction between the AA@BNNPs and polymer matrix, and by the better dispersion of AA@BNNPs within a polymer matrix. The traces of $\mathrm{E}$ and $\tan \delta$ of PVA composites are shown in Figure 5b,c. As illustrated in Figure $5 b$, the glass transition temperatures $\left(T_{\mathrm{g}}\right)$ acquired from corresponding tan $\delta$ peaks of PVA, $8.3 \mathrm{wt} \% \mathrm{~h}-\mathrm{BN} / \mathrm{PVA}$, $8.3 \mathrm{wt} \%$ BNNP/PVA, $8.3 \mathrm{wt} \%$ Glu@BNNP/PVA, $8.3 \mathrm{wt} \%$ Lys@BNNP/PVA were 5.9, 6.6, 7.8, 8.3 and $9.1{ }^{\circ} \mathrm{C}$, respectively. Compared with the $8.3 \mathrm{wt} \%$ Glu@BNNP/PVA and Lys@BNNP/PVA, the $11.3 \mathrm{wt} \%$ Glu@BNNP/PVA and Lys@BNNP/PVA have increased to 8.5 and $9.4{ }^{\circ} \mathrm{C}$. It was concluded that as the Glu@BNNP and Lys@BNNP additional amount increased, the hydrogen-bonding interaction was increased. The $T_{\mathrm{g}}$ of these PVA composites was increased with different fillers, which suggested 
that the increase of intermolecular interactions was caused by hydrogen bonds [27]. Moreover, the storage modulus was in the order of the PVA $<8.3 w \mathrm{t} \% \mathrm{~h}-\mathrm{BN} / \mathrm{PVA}<8.3 \mathrm{wt} \% \mathrm{BNNP} / \mathrm{PVA}<8.3 \mathrm{wt} \%$ Glu@BNNP/PVA < 11.3 wt\% Glu@BNNP/PVA < 8.3 wt\% Lys@BNNP/PVA < 11.3 wt $\%$ Lys@BNNP/PVA (see Figure 5c). These dynamic properties were dependent on the incorporated fillers, including the hydrogen-bonding interaction between -OH groups on PVA chain and -COOH groups originating from AA moieties [25,34].
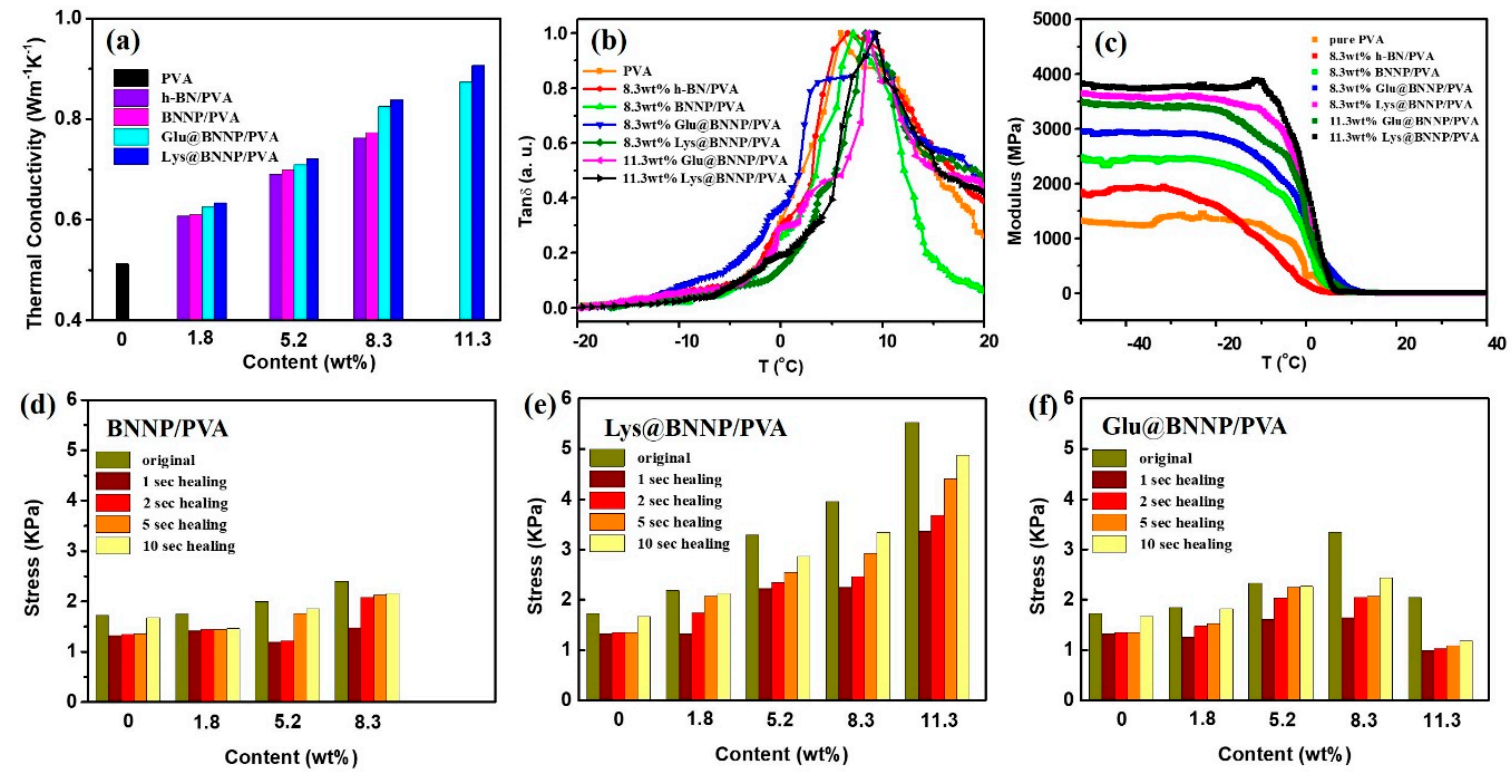

Figure 5. (a) Thermal conductivities of pure PVA, h-BN/PVA, BNNP/PVA, Glu@BNNP/PVA and Lys@BNNP/PVA hydrogel; (b) dynamic mechanical analysis (DMA) tan $\delta$ curves, and (c) storage modulus curves of the PVA hydrogels; $(\mathbf{d}-\mathbf{f})$ original stress and healed stress histogram of BNNP/PVA, Lys@BNNP/PVA hydrogel and Glu@BNNP/PVA hydrogel with different contents of BNNP, Lys@BNNP and Glu@BNNP.

The self-healing experiments route and mechanical measurements [23] of the PVA hydrogel were similar to the previous work. In addition to improving thermal conductivity, the hydrogen-bonding interaction also can enhance the mechanical performance of BNNP/PVA and AA@BNNPs/PVA hydrogel composites, as shown in Figure 5d-f. To further assess their self-healing behaviors [25], the tensile test was carried out to evaluate the healing efficiency. After the hydrogel was cut into two pieces, two parts of the gel were put together and re-contacted for $60 \mathrm{~s}$ with no other stress or outside stimulus applied, and then the healed hydrogel was stretched to test the tensile strength, examples as Figure 4e. Lys@BNNP/PVA and Glu@BNNP/PVA hydrogels both retained excellent self-healing properties, the tensile stress to $88 \%$ for Lys@BNNP/PVA with filling content of $11.3 \mathrm{wt} \%$, and $74 \%$ for Glu@BNNP/PVA with filling content of $8.3 w t \%$. Compared with Glu@BNNP/PVA, the performance of Lys@BNNP/PVA was much better. The reason was that the grafted amino acid moieties of Lys@BNNP were higher than in Glu@BNNP, and there were more hydrogen bonds between the Lys@BNNP/PVA hydrogels. The detailed experimental data (force-displacement curve and stress-strain curve) and process can be found in Figures S4-S11.

\section{Conclusions}

In summary, we have made a demonstration that economical and biobased AAs can be employed as assisting reagents to prepare well-compatible BNNP derivatives via mechanical exfoliation. With this approach, the dispersion state of AA@BNNPs derivatives in water have been effectively stabilized and transferred into a PVA hydrogel matrix. Based on this, PVA-based hydrogel composites have been prepared with Lys@BNNP and Glu@BNNP as fillers. Significant increases for these composites in 
thermal conductivity and mechanical properties resulted, relative to those of h-BN/PVA or BNNP/PVA composites and pure PVA hydrogel. This scalable and environmentally friendly approach offers future scope for facile production of various functional BNNPs.

Supplementary Materials: The following are available online at http:/www.mdpi.com/2079-4991/10/9/1652/s1. Figure S1. (a)-(d) C 1s and O 1s core-level spectra of Lys@BNNP and Glu@BNNP. Figure S2. (a) Low-magnification TEM image of BNNP, (b) high-resolution TEM images of the BNNP. Figure S3. Raman spectroscopy of as-received h-BN, Lys@BNNP and Glu@BNNP. Figure S4. (a) Force-displacement curve of original PVA hydrogel and the healed PVA hydrogels, (b) tensile stress-strain curve of original PVA hydrogel and the healed PVA hydrogels. Figure S5. Force-displacement curve of BNNP/PVA hydrogels with different contents of BNNP $(1.8,5.2$ and $8.3 \mathrm{wt} \%)$ and the healed BNNP/PVA hydrogels (a) $1.8 \mathrm{wt} \%$, (b) $5.2 \mathrm{wt} \%$, (c) $8.3 \mathrm{wt} \%$. Figure S6. Tensile stress-strain curve of $\mathrm{BNNP} / \mathrm{PVA}$ hydrogels with different contents of BNNP $(1.8,5.2$ and $8.3 \mathrm{wt} \%)$, and the healed BNNP/PVA hydrogels (a) $1.8 \mathrm{wt} \%$, (b) $5.2 \mathrm{wt} \%$, (c) $8.3 \mathrm{wt} \%$. Figure S7. Force-displacement curve of Lys@BNNP/PVA hydrogels with different contents of Lys@BNNP $(1.8,5.2,8.3$ and 11.3 wt $\%)$, and the healed Lys@BNNP/PVA hydrogels (a) 1.8 wt $\%$, (b) $5.2 \mathrm{wt} \%$, (c) $8.3 \mathrm{wt} \%$ and (d) $11.3 \mathrm{wt} \%$. Figure S8. Tensile stress-strain curve of Lys@BNNP/PVA hydrogel with different contents of Lys@BNNP (1.8, 5.2, 8.3 and $11.3 \mathrm{wt} \%)$, and the curves of healed Lys@BNNP/PVA hydrogels (a) $1.8 \mathrm{wt} \%$, (b) $5.2 \mathrm{wt} \%$, (c) $8.3 \mathrm{wt} \%$ and (d) $11.3 \mathrm{wt} \%$. Figure S9. Force-displacement curve of original Glu@BNNP/PVA hydrogel with different contents of Glu@BNNP $(1.8,5.2,8.3$ and $11.3 \mathrm{wt} \%)$, and the curves of healed Glu@BNNP/PVA hydrogels (a) $1.8 \mathrm{wt} \%$, (b) $5.2 \mathrm{wt} \%$, (c) $8.3 \mathrm{wt} \%$ and (d) $11.3 \mathrm{wt} \%$. Figure S10. Tensile stress-strain curve of original Glu@BNNP/PVA hydrogel with different contents of Glu@BNNP $(1.8,5.2,8.3$ and $11.3 \mathrm{wt} \%$ ), and the curves of healed Glu@BNNP/PVA hydrogels (a) $1.8 \mathrm{wt} \%$, (b) $5.2 \mathrm{wt} \%$, (c) $8.3 \mathrm{wt} \%$ and (d) $11.3 \mathrm{wt} \%$. Figure S11. The self-healing test process of strip-shaped hydrogel samples. In order to test the rapid self-healing performance of the BN samples, self-healing tests were carried out directly on the electronic universal testing machine. (a) Strip-shaped sample was clamped on the testing machine; (b) the hydrogel is cut into two pieces; (c) the two pieces of the hydrogel are re-contacted; (d) the hydrogel can self-heal automatically after contacting for $1 \mathrm{~s}, 2 \mathrm{~s}, 5 \mathrm{~s}$ or $10 \mathrm{~s}$ at room temperature; (e) the healed hydrogel is being tensile tested; and (f) end of healed hydrogel tensile test.

Author Contributions: Conceptualization, N.Y., formal analysis, N.Y. and H.J., investigation, N.Y. and H.J., resources, X.Z. and B.L., data curation, N.Y., writing-original draft preparation, N.Y., writing-review and editing, X.Z., Y.Z. and X.J., visualization, X.Q. and X.Z., supervision, X.Z., Y.Z. and B.L., project administration, X.Z., funding acquisition, X.Z. All authors have read and agreed to the published version of the manuscript.

Funding: This work was supported by the National Natural Sciences Foundation of China (Grant No. 51673192), Natural Sciences Foundation of Tianjin (Grant No. 18JCYBJC43000), and Natural Sciences Foundation of Hebei Province (Grant No. B2020202042 and B2019202153).

Conflicts of Interest: All authors declare no competing financial interest.

\section{References}

1. Xiao, F.; Naficy, S.; Casillas, G.; Khan, M.H.; Katkus, T.; Jiang, L.; Liu, H.; Li, H.; Huang, Z. Edge-Hydroxylated Boron Nitride Nanosheets as an Effective Additive to Improve the Thermal Response of Hydrogels. Adv. Mater. 2015, 27, 7196-7203. [CrossRef]

2. Wang, M.; Jang, S.K.; Jang, W.J.; Kim, M.; Park, S.Y.; Kim, S.W.; Kahng, S.J.; Choi, J.Y.; Ruoff, R.S.; Song, Y.J.; et al. A Platform for Large-Scale Graphene Electronics-CVD Growth of Single-Layer Graphene on CVD-Grown Hexagonal Boron Nitride. Adv. Mater. 2013, 25, 2746-2752. [CrossRef] [PubMed]

3. Yang, W.; Chen, G.; Shi, Z.; Liu, C.C.; Zhang, L.; Xie, G.; Cheng, M.; Wang, D.; Yang, R.; Shi, D.; et al. Epitaxial growth of single-domain graphene on hexagonal boron nitride. Nat. Mater. 2013, 12, 792-797. [CrossRef] [PubMed]

4. Weng, Q.; Wang, X.; Wang, X.; Bando, Y.; Golberg, D. Functionalized hexagonal boron nitride nanomaterials: Emerging properties and applications. Chem. Soc. Rev. 2016, 45, 3989-4012. [CrossRef] [PubMed]

5. Zhi, B.C.; Bando, Y.; Terao, T.; Tang, C.; Kuwahara, H.; Golborg, D. Towards Thermoconductive, Electrically Insulating Polymeric Composites with Boron Nitride Nanotubes as Fillers. Adv. Funct. Mater. 2009, 19, 1856-1862. [CrossRef]

6. Wang, X.B.; Weng, Q.; Wang, X.; Li, X.; Zhang, J.; Liu, F.; Jiang, X.F.; Guo, H.; Xu, N.; Golborg, D.; et al. Biomass-Directed Synthesis of $20 \mathrm{~g}$ High-Quality Boron Nitride Nanosheets for Thermoconductive Polymeric Composites. ACS Nano 2014, 8, 9081-9088. [CrossRef]

7. Sainsbury, T.; Satti, A.; Mar, P.; Wang, Z.; McGovern, I.; Gun'ko, Y.K.; Coleman, J. Oxygen Radical Functionalization of Boron Nitride Nanosheets. J. Am. Chem. Soc. 2012, 134, 18758-18771. [CrossRef] 
8. Wang, T.; Ou, D.; Liu, H.; Jiang, S.; Huang, W.; Fang, X.; Chen, X.; Lu, M. Thermally Conductive Boron Nitride Nanosheet Composite Paper as a Flexible Printed Circuit Board. ACS App. Nano Mater. 2018, 1, 1705-1712. [CrossRef]

9. Nie, X.; Li, G.; Jiang, Z.; Li, W.; Ouyang, T.; Wang, J. Co-Solvent Exfoliation of Hexagonal Boron Nitride: Effect of Raw Bulk Boron Nitride Size and Co-Solvent Composition. Nanomaterials 2020, 10, 1035. [CrossRef]

10. Xing, L.; Hu, C.; Zhang, Y.; Wang, X.; Shi, L.; Ran, R. A mechanically robust double-network hydrogel with high thermal responses via doping hydroxylated boron nitride nanosheets. J. Mater. Sci. 2019, 54, 3368-3382. [CrossRef]

11. Lee, D.; Lee, S.; Byun, S.; Paik, K.; Song, S.H. Novel dielectric BN/epoxy nanocomposites with enhanced heat dissipation performance for electronic packaging. Compos. Part A Appl. Sci. Manuf. 2018, 107, 217-223. [CrossRef]

12. Rousseas, M.; Goldstein, A.P.; Mickelson, W.; Worsley, M.A.; Woo, L.; Zettl, A. Synthesis of Highly Crystalline $\mathrm{sp}^{2}$-Bonded Boron Nitride Aerogels. ACS Nano 2013, 7, 8540-8546. [CrossRef] [PubMed]

13. Yin, J.; Li, X.; Zhou, J.; Guo, W. Ultralight Three-Dimensional Boron Nitride Foam with Ultralow Permittivity and Superelasticity. Nano Lett. 2013, 13, 3232-3236. [CrossRef]

14. Lee, D.; Lee, B.; Park, K.H.; Ryu, H.J.; Jeon, S.; Hong, S.H. Scalable Exfoliation Process for Highly Soluble Boron Nitride Nanoplatelets by Hydroxide-Assisted Ball Milling. Nano Lett. 2015, 15, 1238-1244. [CrossRef] [PubMed]

15. Lin, Y.; Williams, T.V.; Cao, W.; Elsayed-Ali, H.E.; Connell, J.W. Defect Functionalization of Hexagonal Boron Nitride Nanosheets. J. Phys. Chem. C 2010, 114, 17434-17439. [CrossRef]

16. Lei, W.; Mochalin, V.N.; Liu, D.; Qin, S.; Gogotsi, Y.; Chen, Y. Boron nitride colloidal solutions, ultralight aerogels and freestanding membranes through one-step exfoliation and functionalization. Nat. Commun. 2015, 6, 1-8. [CrossRef]

17. Lin, Y.; Williams, T.V.; Connell, J.W. Soluble, Exfoliated Hexagonal Boron Nitride Nanosheets. J. Phys. Chem. Lett. 2009, 1, 277-283. [CrossRef]

18. Xie, S.Y.; Wang, W.; Fernando, K.A.S.; Wang, X.; Lin, Y.; Sun, Y.P. Solubilization of boron nitride nanotubes. Chem. Commun. 2005, 29, 3670-3672. [CrossRef]

19. Liu, J.; Gu, J.; Luo, J.; Wang, S.; Zhang, H.; Tao, Y. Controlled Synthesis of Thermoresponsive Polymers Derived from L-Lysine, a Biorenewable Resource. J. Polym. Sci. Pol. Chem. 2019, 57, 862-868. [CrossRef]

20. Pati, D.; Shaikh, A.Y.; Hotha, S.; Gupta, S.S. Synthesis of glycopolypeptides by the ring opening polymerization of O-glycosylated-a-amino acid N-carboxyanhydride (NCA). Polym. Chem. 2011, 2, 805-811. [CrossRef]

21. Chen, C.; Wang, Z.; Li, Z. Thermoresponsive Polypeptides from Pegylated Poly-L-glutamates. Biomacromolecules 2011, 18, 2859-2863. [CrossRef] [PubMed]

22. Liu, S.; Zhang, X.; Li, M.; Ren, X.; Tao, Y. Precision Synthesis of Sustainable Thermoplastic Elastomers from Lysine-Derived Monomers. J. Polym. Sci. Pol. Chem. 2017, 52, 349-355. [CrossRef]

23. Chen, W.P.; Hao, D.Z.; Hao, W.J.; Guo, X.L.; Jiang, L. Hydrogel with Ultrafast Self-Healing Property Both in Air and Underwater. ACS Appl. Mater. Interfaces 2018, 10, 1258-1265. [CrossRef] [PubMed]

24. Jun, H.; Li, G.H.; Yang, N.; Qin, L.L.; Grami, M.E.; Zhang, Q.X.; Wang, N.Y.; Qu, X.W. Preparation and characterization of surface modified boron nitride epoxy composites with enhanced thermal conductivity. RSC Adv. 2014, 4, 44282-44290.

25. Shao, C.; Chang, H.; Wang, M. High-Strength, Tough, and Self-Healing Nanocomposite Physical Hydrogels Based on the Synergistic Effects of Dynamic Hydrogen Bond and Dual Coordination Bonds. ACS Appl. Mater. Interfaces 2017, 9, 28305-28318. [CrossRef]

26. Xiao, Q.; Zhan, C.; You, Y.; Tong, L.; Wei, R.; Liu, X. Preparation and thermal conductivity of copper phthalocyanine grafted boron nitride nanosheets. Mater. Lett. 2018, 227, 33-36. [CrossRef]

27. Wu, X.; Liu, H.; Tang, Z.; Guo, B. Scalable fabrication of thermally conductive elastomer/boron nitride nanosheets composites by slurry compounding. Compos. Sci. Technol. 2016, 123, 179-186. [CrossRef]

28. Lei, W.; Liu, D.; Chen, Y. ighly Crumpled Boron Nitride Nanosheets as Adsorbents: Scalable Solvent-Less Production. Adv. Mater. Interfaces 2015, 2, 1400529. [CrossRef]

29. Wang, Y.; Cao, Z.; Wu, J.; Ma, C.; Qiu, C.; Zhao, Y.; Shao, F.; Wang, H.; Zheng, J.; Huang, G. Mechanically robust, ultrastretchable and thermal conducting composite hydrogel and its biomedical applications. Chem. Eng. J. 2019, 360, 231-242. [CrossRef] 
30. Lin, H.; Mehra, N.; Li, Y.; Zhu, J. Graphite oxide/boron nitride hybrid membranes: The role of cross-plane laminar bonding for a durable membrane with large water flux and high rejection rate. J. Membr. Sci. 2020, 593, 117401. [CrossRef]

31. Lin, Z.; Mcnamara, A.; Liu, Y.; Moon, K.; Wong, C. Exfoliated hexagonal boron nitride-based polymer nanocomposite with enhanced thermal conductivity for electronic encapsulation. Compos. Sci. Technol. 2014, 90, 123-128. [CrossRef]

32. Shahil, K.M.F.; Balandin, A.A. Graphene-Multilayer Graphene Nanocomposites as Highly Efficient Thermal Interface Materials. Nano Lett. 2012, 12, 861-867. [CrossRef] [PubMed]

33. Wu, K.; Fang, J.; Ma, J.; Huang, R.; Chai, S.; Chen, F.; Fu, Q. Achieving a Collapsible, Strong, and Highly Thermally Conductive Film Based on Oriented Functionalized Boron Nitride Nanosheets and Cellulose Nanofiber. ACS Appl. Mater. Interfaces 2017, 9, 30035-30045. [CrossRef] [PubMed]

34. Jing, L.; Li, H.; Tay, R.Y.; Sun, B.; Tsang, S.H.; Cometto, O.; Lin, J.; Teo, E.H.T.; Tok, A.I.Y. Biocompatible Hydroxylated Boron Nitride Nanosheets/Poly(vinyl alcohol) Interpenetrating Hydrogels with Enhanced Mechanical and Thermal Responses. ACS Nano 2017, 11, 3742-3751. [CrossRef] [PubMed]

(C) 2020 by the authors. Licensee MDPI, Basel, Switzerland. This article is an open access article distributed under the terms and conditions of the Creative Commons Attribution (CC BY) license (http://creativecommons.org/licenses/by/4.0/). 